\title{
A smartphone-supported weight loss program: design of the ENGAGED randomized controlled trial
}

\author{
Christine A Pellegrini ${ }^{*}$, Jennifer M Duncan ${ }^{1}$, Arlen C Moller ${ }^{1}$, Joanna Buscemi ${ }^{1}$, Alyson Sularz ${ }^{1}$, Andrew DeMott ${ }^{1}$,
} Alex Pictor ${ }^{1}$, Sherry Pagoto ${ }^{2}$, Juned Siddique ${ }^{1}$ and Bonnie Spring ${ }^{1}$

\begin{abstract}
Background: Obesity remains a major public health challenge, demanding cost-effective and scalable weight management programs. Delivering key treatment components via mobile technology offers a potential way to reduce expensive in-person contact, thereby lowering the cost and burden of intensive weight loss programs. The ENGAGED study is a theory-guided, randomized controlled trial designed to examine the feasibility and efficacy of an abbreviated smartphone-supported weight loss program.

Methods/design: Ninety-six obese adults (BMI $30-39.9 \mathrm{~kg} / \mathrm{m}^{2}$ ) will be randomized to one of three treatment conditions: (1) standard behavioral weight loss (STND), (2) technology-supported behavioral weight loss (TECH); or (3) self-guided behavioral weight loss (SELF). All groups will aim to achieve a $7 \%$ weight loss goal by reducing calorie and fat intake and progressively increasing moderate intensity physical activity to 175 minutes/week. STND and TECH will attend 8 group sessions and receive regular coaching calls during the first 6 months of the intervention; SELF will receive the Group Lifestyle Balance Program DVD's and will not receive coaching calls. During months 1-6, TECH will use a specially designed smartphone application to monitor dietary intake, body weight, and objectively measured physical activity (obtained from a Blue-tooth enabled accelerometer). STND and SELF will self-monitor on paper diaries. Linear mixed modeling will be used to examine group differences on weight loss at months 3, 6, and 12. Self-monitoring adherence and diet and activity goal attainment will be tested as mediators.
\end{abstract}

Discussion: ENGAGED is an innovative weight loss intervention that integrates theory with emerging mobile technologies. We hypothesize that TECH, as compared to STND and SELF, will result in greater weight loss by virtue of improved behavioral adherence and goal achievement.

Trial registration: NCT01051713

Keywords: Weight loss, Technology, Physical activity

\section{Background}

Obesity remains a pressing public health problem. Approximately $36 \%$ of adults in the United States are obese (Body Mass Index $(\mathrm{BMI}) \geq 30 \mathrm{~kg} / \mathrm{m}^{2}$ ) [1]. Globally, the World Health Organization estimates that approximately 500 million adults were obese in 2008 [2]. Obesity

\footnotetext{
* Correspondence: c-pellegrini@northwestern.edu

${ }^{1}$ Current Address: Department of Preventive Medicine, Feinberg School of Medicine, Northwestern University, 680 N. Lake Shore Drive, Suite 1400,

Chicago, IL 60611, USA

Full list of author information is available at the end of the article
}

heightens the risk of a number of diseases, including type 2 diabetes, cardiovascular disease, obstructive sleep apnea, gallbladder disease, osteoarthritis, and several forms of cancer [3-6]. Consequently, obesity creates significant economic burden: U.S. medical costs associated with obesity were $\$ 147$ billion in 2008 [7].

The Diabetes Prevention Program (DPP) intensive lifestyle intervention, which targets decreasing caloric and fat intake and increasing moderate-to-vigorous intensity physical activity, has been successful in producing clinically significant weight loss and reducing the incidence of

\section{Biomed Central}


diabetes in pre-diabetic adults [8]. In spite of its efficacy for weight loss and improving cardiometabolic outcomes, the DPP lifestyle intervention has not been widely adopted in its original form, primarily because it includes sixteen face-to-face sessions and sixteen to twenty-four optional maintenance meetings. This amount and type of contact is considered too burdensome and expensive to be a sustainable program [9]. In an attempt to increase the reach of this program, the DPP protocol has been translated for implementation in real-world settings [10] such as in a community YMCA [11] and a community-based diabetes education program led by community health workers [12]. Significant weight losses were produced ranging from $6.0-7.3 \%$ in both studies. However these programs may still have limited reach due to the 16-24 weekly group sessions required during months $1-6$ and additional maintenance meetings during months $7-12[11,12]$. To date, studies that have reduced treatment intensity by reducing the number of in-person sessions have resulted in poorer weight loss outcomes [13]. Therefore, the still unmet challenge of DPP implementation is how to reduce inperson contact without eliminating features considered essential for successful weight loss: social support, accountability, and feedback.

\section{Technology and weight loss interventions}

Technology-based interventions hold great promise as a mechanism for disseminating efficacious weight loss programs to a broader portion of the population via a cost-effective, scalable platform [14]. Many different technologies have been used in weight loss interventions including PDAs $[15,16]$, cell phone text messages $[17,18]$ and wearable physical activity monitors $[19,20]$. However, Internet-based interventions have received the most empirical attention to date [21]. Internet-based weight loss treatment findings have been mixed, and those programs that have achieved the highest weight losses have also included interactive tools, tailored feedback to participants, and in-person contact [21-24]. Programs that are solely internet-based however, do not allow for the same level of interactivity, social support, and feedback that occurs during in-person interventions.

New generation handheld technologies have the potential to overcome many of the limitations of internetbased health behavior change interventions [25]. Mobile phones are becoming ubiquitous: $46 \%$ of Americans own a smartphone, with rates even higher among minority groups [26]. Smartphones provide an unprecedented opportunity for frequent and interactive feedback, tailored email or text reminders, and immediate access to social support from peers and coaches. Interactive smartphone applications can be used as a decision support tool to provide timely information on health behavior decisions occurring in real-time. These technological advances afford a promising opportunity to preserve the key components of intensive in-person treatment approaches (i.e., regular social support, accountability, and frequent feedback) while requiring fewer in-person sessions, thus, lowering the cost and broadening the accessibility of treatment. To test the feasibility and efficacy of this mobile technology-supported strategy, we have developed the E-Networks Guiding Adherence to Goals in Exercise and Diet (ENGAGED) study, a randomized controlled trial (RCT) that uses a theory-guided, technologysupported weight loss program.

\section{Theoretical framework}

The conceptual basis for the ENGAGED study is Carver and Scheier's control systems theory (CST) of selfregulation [27]. CST posits that self-regulation can be understood in terms of feedback loops, wherein people: (1) set a goal, (2) self-monitor their current status to identify discrepancy from a goal, and then (3) modify their behavior to reduce perceived discrepancy. From a CST perspective, DPP's efficacy can be attributed to helping participants (1) set appropriate caloric intake and physical activity goals, (2) self-monitor weight, eating behavior, and activity, and (3) adjust behaviors to reach the targets. According to CST, the core challenge in weight loss is aligning diet and activity with goals. Most weight loss efforts lose because traditional paper and pencil self-monitoring of diet and activity fails to provide salient, timely decision support and accurate feedback.

Using CST as a guiding framework, ENGAGED will develop a persuasive, interactive smartphone application designed improve the saliency, timeliness, and accuracy with which participants receive feedback on their daily dietary and activity goals. Participants will monitor dietary intake and wear a Bluetooth-enabled accelerometer to receive real-time feedback on objectively measured physical activity. Persuasively designed goal thermometers on the application will display current behavior and goals to highlight behavior-goal discrepancies. We posit based on CST that the experience of receiving consistent and immediate feedback from the ENGAGED technology should reinforce adherence to self-monitoring and healthy behavior change, both during and after in-person treatment ends.

\section{Social networks, peer support, and weight loss}

Research on social networks and weight has elicited promising results. Christakis and Fowler [28] reported that obesity can spread virally through social networks. Relatedly, other findings suggest that including friends and family as co-participants in weight loss treatment improves outcomes [29]. However, not all overweight 
and obese adults have family or friends willing to join and/or support them in their weight loss efforts. Thus, additional strategies to provide peer support need to be identified. Social networking tools have gained popularity on many online commercial weight management programs, and preliminary evidence indicates that technology-supported social networking can be beneficial. Krukowski et al. [30] found that use of social networking features in an online weight management program was positively associated with weight loss maintenance.

The ENGAGED study will utilize technology that enhances social networking capabilities on a smartphone application to promote the development and maintenance of a positive social network. The application will allow teammates who have been incentivized to help each other via a group weight loss competition to view each others' adherence to daily self-monitoring goals. Additionally, the application allows team members to communicate with each other via private message board or peer-to-peer messaging. By capitalizing on team-based performance incentives and enabling team building through the ENGAGED social networking technology, we aim to extend support, encouragement, and accountability for behavioral change, between and after in-person group sessions.

\section{Study aims}

Guided by CST and social networks research, the ENGAGED study will evaluate the feasibility and efficacy of a technology-supported weight loss program for obese adults. Specifically, the primary aim of the ENGAGED study is to conduct a three-group randomized controlled trial testing the efficacy of the ENGAGED technologysupported behavioral weight loss program, as compared to the same program delivered using standard paper and pencil self-monitoring, and self-guided behavioral weight loss supported by participants receiving DVD's depicting DPP treatment. Primary outcomes include: (1) weight loss and (2) behavioral adherence, which is operationalized by (a) self-monitoring of diet and activity and (b) attainment of diet and activity goals. As a secondary outcome, the time spent administering the intervention by lifestyle coaches will also be examined to assess intervention costs. The second aim of the ENGAGED study is to test whether the weight loss in the technology-supported condition is mediated by behavioral adherence to self-monitoring and achievement of diet and activity goals.

We hypothesize that using a theory-guided, technology-supported weight loss treatment will result in clinically significant weight loss with fewer in-person sessions by preserving regular social support, accountability, and feedback via mobile technology. Further, we hypothesize that technology-supported weight loss treatment will result in greater weight loss compared to the traditional paper self-monitoring approach.

\section{Methods \\ Study design}

The research design is a $3 \times 4$ factorial with one between subjects factor (treatment condition) with three levels and one within subjects factor (time) with four levels (baseline, 3-, 6-, and 12-months). Participants will be randomized to one of three groups: a) Standard behavioral weight loss program (STND); b) technologysupported behavioral weight loss program (TECH); or c) self-guided behavioral weight loss program (SELF).

\section{Eligibility}

A total of 96 participants who meet the inclusion and exclusion criteria listed in Table 1 will be recruited in two cohorts. Participants will be between $18-60$ years old with a body mass index (BMI) between $30-40 \mathrm{~kg} / \mathrm{m}^{2}$, weight stable for the past 6 months, not currently enrolled in a formal weight loss program or taking weight loss medications, and be willing to self-monitor and wear an accelerometer daily for 6 months. Participants will be excluded if they have any unstable medical conditions, are currently pregnant, or taking any medications known to influence weight. See Table 1 for a complete list of exclusion criteria.

\section{Recruitment and screening process}

All study procedures are approved by the Institutional Review Board at Northwestern University. Participants will be recruited via flyers and advertisements in local newspapers and on public transportation. Interested candidates will be directed to a study website which includes study-related information and an online screening questionnaire to assess initial eligibility. Figure 1 illustrates the screening process.

\section{Online screening}

Participants will complete an initial online consent process prior to completing the online screener, which assesses height and weight, physical activity readiness, group session availability, and exclusionary weight loss practices (i.e. participation in a weight loss program or use of weight loss medications). Study staff will contact eligible participants within 1-4 days of completing an online screener to conduct a telephone screen.

\section{Telephone screening}

The telephone screen will review details of the study and review additional eligibility criteria including health status and medication use. Eligible and interested participants will be invited to attend a study orientation 
Table 1 ENGAGED inclusion and exclusion criteria

\begin{tabular}{|c|c|}
\hline Inclusion & criteria \\
\hline & - BMI $30.0-39.9 \mathrm{~kg} / \mathrm{m}^{2}$ \\
\hline & - 18-60 years old \\
\hline & - Interested in losing weight through diet and physical activity changes \\
\hline & - Weight stable over the past 6 months \\
\hline & - Not receiving concurrent weight loss treatment \\
\hline & - Willing to self-monitor (on smartphone or paper) and wear an accelerometer daily for 6 months \\
\hline & - Able to attend all assessments and group sessions \\
\hline & - Have or be willing to learn basic technology usage skills \\
\hline Exclusion & criteria \\
\hline & $\begin{array}{l}\text { - Has one or more of the following unstable medical conditions: uncontrolled hypertension or diabetes, angina pectoris, myocardial } \\
\text { infarction, plantar fasciitis, transient ischemic attack, cancer undergoing active treatment, or cerebrovascular accident within the past } 6 \\
\text { months }\end{array}$ \\
\hline & - Has insulin-dependent diabetes, Crohn's Disease, obstructive sleep apnea requiring use of a CPAP, plantar fasciitis \\
\hline & - Requires use of an assistive device for mobility \\
\hline & - Has been hospitalized for a psychiatric disorder in the past 5 years \\
\hline & - Contraindications for moderate intensity physical activity \\
\hline & - Currently taking any weight-loss medications or committed to following an incompatible dietary regimen \\
\hline & - Currently pregnant, trying to get pregnant, or lactating \\
\hline & - Current bulimia nervosa or binge eating disorder \\
\hline & - Current substance or alcohol or dependence \\
\hline & - Current attention deficit hyperactivity disorder or major depressive disorder \\
\hline & - Currently taking medication(s) known to cause weight gain (i.e. Prednisone, Depakote, Diebeta, Diabinese, Cardura, Inderal, Zyprexa) \\
\hline & - Expresses active suicidal ideation \\
\hline
\end{tabular}

session and will be instructed to begin a one-week run-in period of dietary self-monitoring. If participants indicate a health condition such as type II diabetes or hypertension, medical clearance from a physician will be required prior to the pretreatment session in order to continue participation.

\section{In-person orientation session}

During the orientation session, complete details of the study will be given and an equipoise induction will be performed to ensure that participants are aware of the pros and cons of the treatment conditions and to minimize the risk of differential attrition [31]. Participant questions will be answered and informed consent will be obtained from interested participants. Consented participants will complete the final in-person screening and baseline assessment, which includes assessing blood pressure and anthropometric measurements. In addition, participants will complete a smartphone competency task to evaluate their ability to use basic functions of a smartphone after instruction and will complete a brief interview to assess: binge eating disorder, bulimia nervosa, substance abuse and dependence, attention deficit hyperactivity disorder, and major depressive disorder. Eligible participants will also complete baseline questionnaires and wear an accelerometer for 10 days to measure current activity levels.

\section{Pre-treatment session}

At the pretreatment session, participants will return the accelerometer, meet with a dietician to complete a dietary recall, and engage in a brief treadmill activity. During the treadmill activity, participants will walk on a treadmill at a moderate intensity while reviewing ratings of perceived exertion to become familiar with the physiological signs of physical activities at this intensity.

\section{Randomization}

Eligible participants will be scheduled for a group time and assigned to a team with seven other participants, all of whom will be randomized to the same condition. Group randomization will be completed by a statistician, and neither participants, nor coaches, will find out group assignment until the first group session. The intervention timeline is presented in Figure 2.

\section{Standard weight loss program (STND)}

STND participants will be given a goal of $7 \%$ weight loss to be achieved through changes in diet and activity, similar to the DPP lifestyle intervention [32]. Based on baseline weight, participants will be given daily calorie 


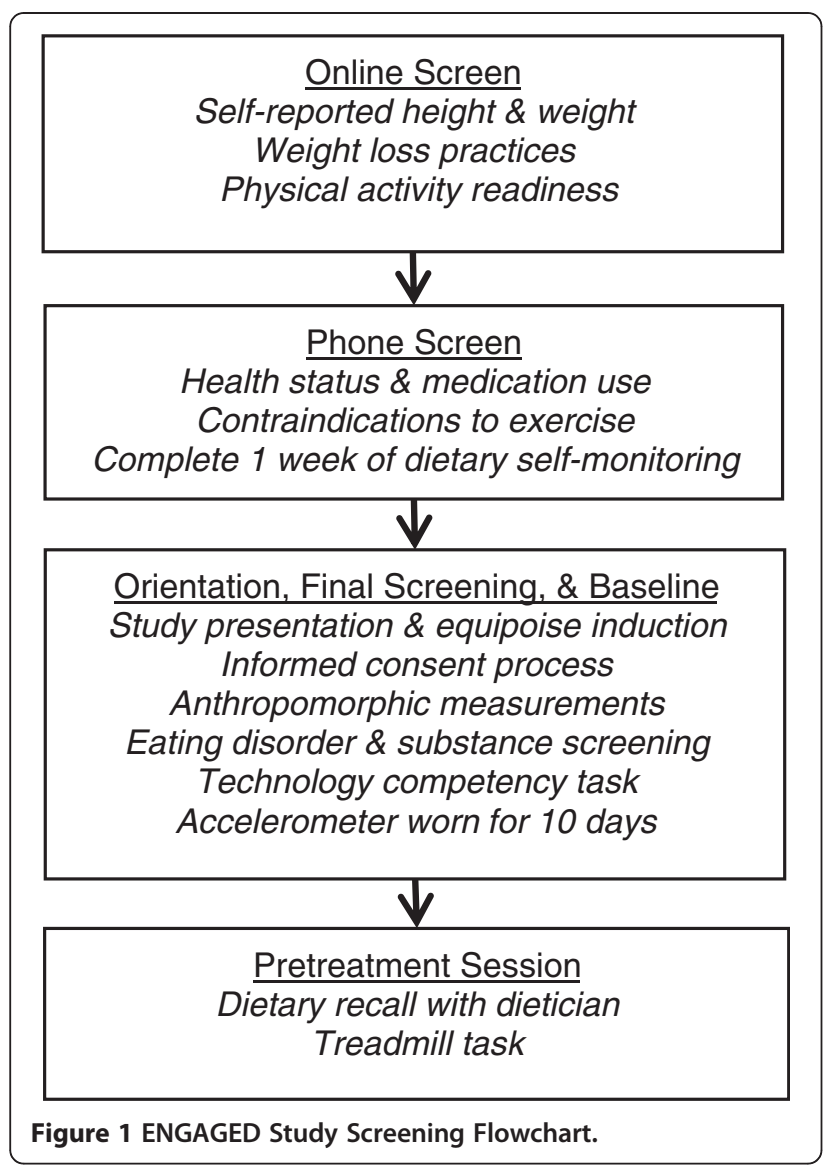

goal between $1200-2000 \mathrm{kcal} /$ day and a $25 \%$ fat gram goal between $32-55$ grams/day. These goals are designed to produce a one to two pound weight loss per week. Participants will also be given a weekly physical activity goal, beginning with 45 minutes of moderate intensity physical activity per week and progressing weekly to 175 minutes per week. Participants will be provided with "The Complete Book of Food Counts" [33] and paper Keeping Track diaries during months $1-6$ to facilitate self-monitoring of dietary intake, physical activity, and body weight.

The original sixteen session DPP protocol will be condensed to eight group sessions (see Table 2) and, similar to the Look AHEAD trial [34], group sessions will be combined with individual telephone coaching to increase social support and reduce costs [35]. Group sessions will run 90 minutes in duration and be held weekly for the first eight weeks of the study. Each group session will begin with a private weigh-in and participants will submit their Keeping Track diary. All group sessions will be led by a trained lifestyle interventionist and will include setting an agenda, reviewing homework and self-monitoring, presentation of new material, group discussions, and assignment of homework. See Table 2 for a list of session topics. All group sessions will end with an optional supervised group walking session.

In addition to in-person group sessions, STND participants will receive individual coaching calls. Coaching calls will be scheduled on a weekly basis during the initial eight weeks of the study and monthly during months 3-6. Coaches will review submitted Keeping Track diaries and determine the participant's level of achievement for each goal using standardized adherence criteria for the study. Coaching calls will last approximately 10-15 minutes and follow a structured script aiming to reinforce success and problem solve around barriers to change. Four content areas will be covered during each call: (1) weight loss, (2) adherence to dietary and physical activity self-monitoring, (3) attainment of diet and activity goals, and (4) goal setting.

\section{Technology-supported weight loss program (TECH)}

Similar to STND, TECH will attend eight in-person group sessions, receive regular coaching calls, and follow the same weight, physical activity, and dietary goals. However rather than paper Keeping Track diaries, TECH will be provided with a study smartphone, ENGAGED application, and an accelerometer to use during the first 6 months of the intervention. In addition to covering the topics discussed during the first group meeting (Table 2), TECH will be instructed on how to self-monitor dietary intake, use the accelerometer, and communicate virtually with their teammates and coach using the ENGAGED application. Time-stamped dietary and physical activity data will be continuously downloaded to a secure server, and participant data will be summarized automatically on the secure coaching application and website, allowing coaches to have up-to-date access to participant data, enabling corrective or supportive feedback to be given via text message or during scheduled coaching calls.

\section{ENGAGED technology}

The ENGAGED technology includes a study smartphone $\left(\right.$ Motorola Droid $\left.{ }^{\mathrm{m}}\right)$, pre-loaded with the ENGAGED application, and an accelerometer (SHIMMER $\left.{ }^{\mathrm{m}}\right)$. TECH participants will use the ENGAGED application, which contains the CalorieKing ${ }^{\circledR}$ food database, a comprehensive nutritional source containing over 50,000 food entries, to self-monitor daily dietary intake. Goal thermometers will display participant's goal and actual amount of calories and fat grams consumed (Figure 3). The ENGAGED application also includes a Team tab that allows participants to view team members' adherence to self-monitoring and accelerometer usage. In addition, the Team tab on the application includes a message board and peer-to-peer messaging to facilitate communication and support among teammates. 


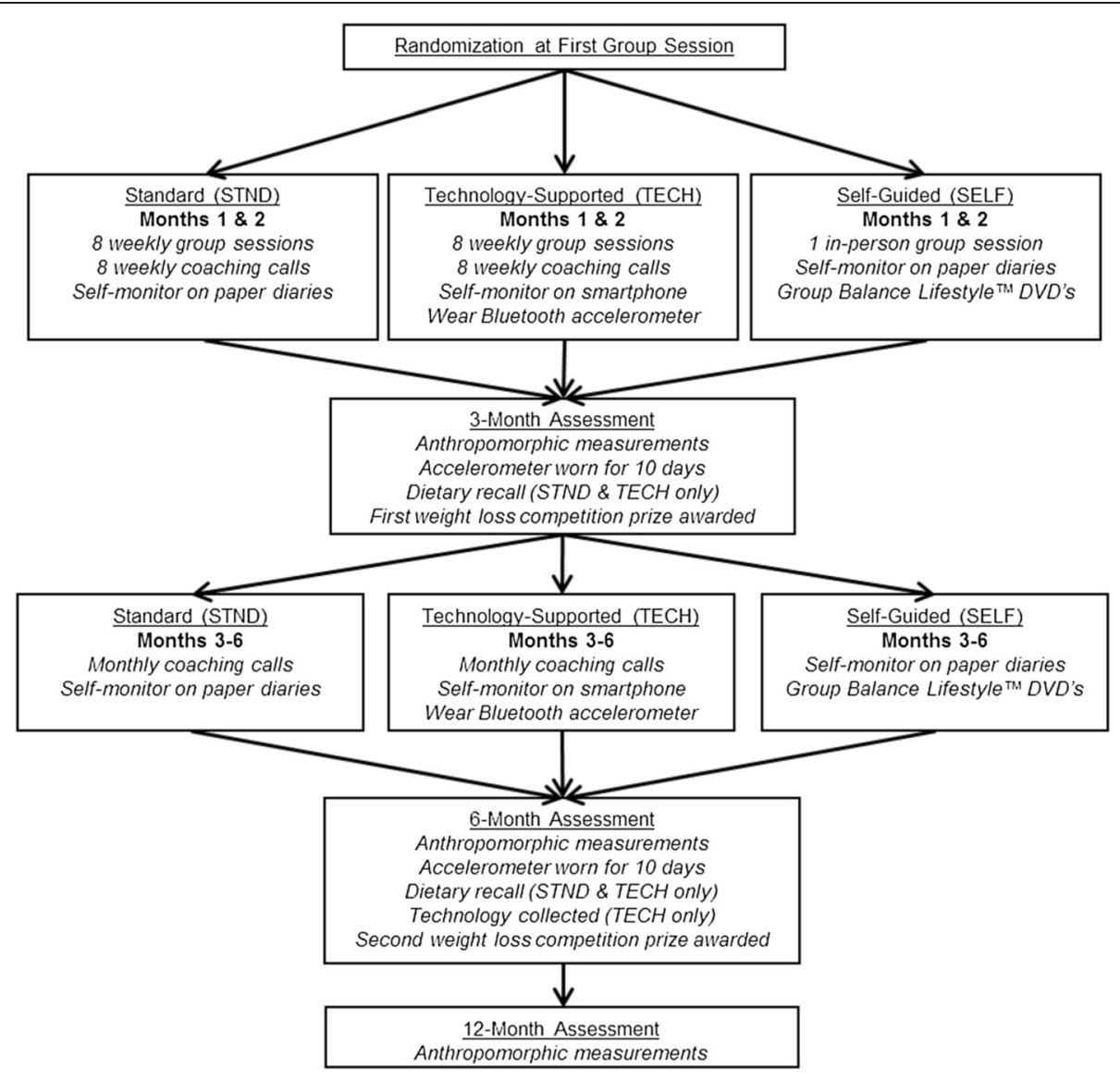

Figure 2 ENGAGED Intervention Timeline.

Participants will also wear an accelerometer that connects via Bluetooth to the smartphone to provide realtime objective data on the accumulation of moderate-tovigorous intensity physical activity. In the event

Table 2 ENGAGED Study Group Session Topics

\begin{tabular}{cl}
\hline Session & Topics covered \\
\hline 1 & Getting Started With Activity and Healthy Eating \\
\cline { 2 - 2 } & Be a Fat Detective \\
\hline 2 & Three Ways to Eat Less Fat \\
\cline { 2 - 2 } & Four Keys to Healthy Eating Out \\
\hline 3 & Take Charge of What's Around You: Making Cues Work for You \\
\hline 4 & Move Those Muscles and Be Active As A Way of Life! \\
\hline 5 & You Can Manage Stress \\
\hline 6 & Talk Back to Negative Thoughts \\
\cline { 2 - 2 } & Problem Solving \\
\hline 7 & Healthy Eating \\
\hline 8 & The Slippery Slope of Lifestyle Change \\
\cline { 2 - 2 } & Wump Start Your Activity Plan to Stay Motivated \\
\hline
\end{tabular}

participants engage in an activity not captured by the accelerometer (i.e. cycling, swimming), users may manually input physical activity.

\section{Self-guided behavioral weight loss program (SELF)}

SELF will attend one 60-minute in-person group session during but will not attend any additional group sessions nor receive coaching calls throughout the intervention. Similar to STND, SELF will be given "The Complete Book of Food Counts" [33] and paper Keeping Track diaries. Rather than attend in-person group sessions, SELF will receive three Group Lifestyle Balance ${ }^{\mathrm{Tm}}$ (GLB) Program DVDs [36] that contain 12 group sessions adapted from the original DPP curriculum as well as the corresponding GLB participant handouts. SELF will be given their individual $7 \%$ weight loss goals and instructed to watch the DVDs to get their calorie and fat gram goals.

\section{Team weight loss competition}

In order to encourage members of each weight loss team to support each other's behavioral adherence and weight loss, a team weight loss competition will be held at 


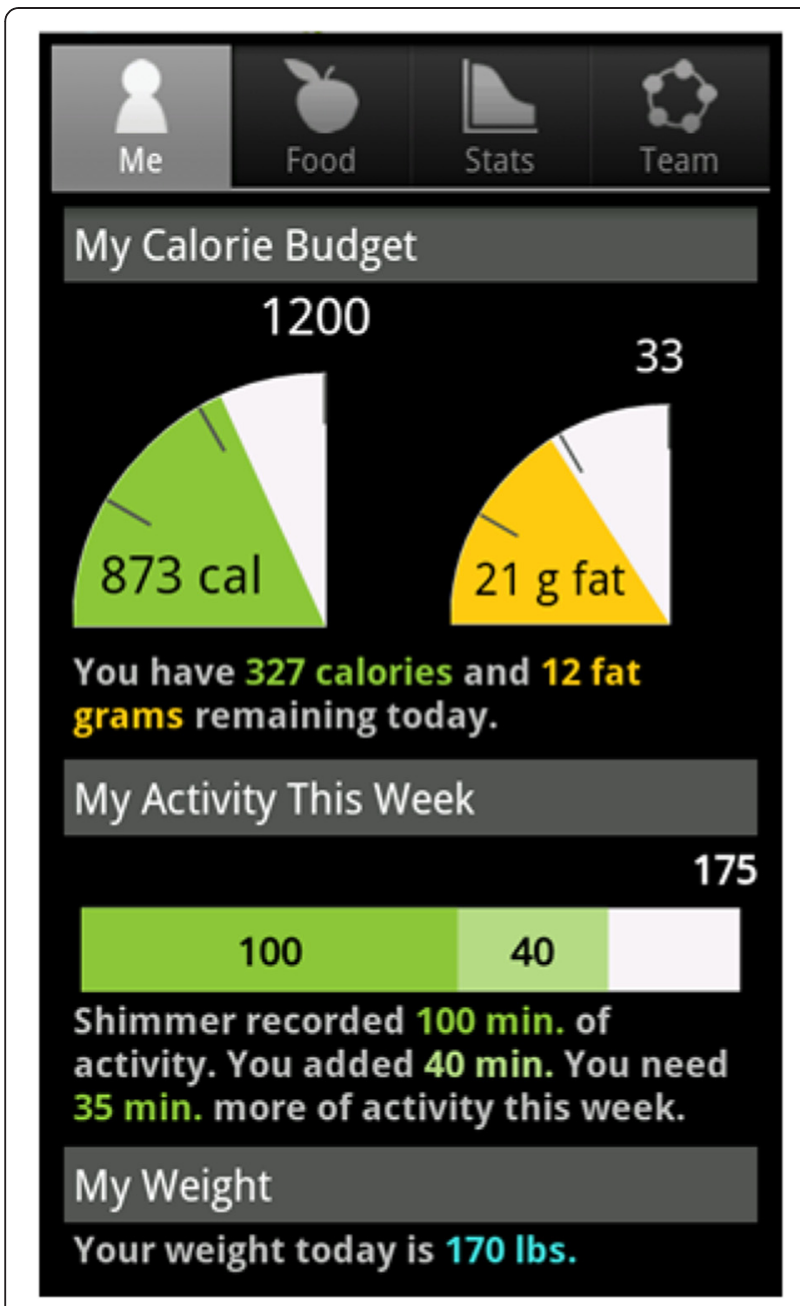

Figure 3 Screen shot of ENGAGED smartphone application.
3- and 6-months during each cohort. The team with the greatest weight loss percentage at each time point will win $\$ 400$, to be divided evenly among the group members ( $\$ 50$ per team member).

\section{Treatment fidelity}

Fidelity checklists will be prepared for group and telephone coaching sessions. Checklists for each session will specify a) good counseling practices; b) prescribed session content; and c) proscribed session content. Coaches will undergo training to become familiarized with the modified DPP protocol and to role-play potential conflicts and situations that may arise during group and telephone sessions. Study staff will also attend weekly clinical meetings to discuss any participant conflicts that may arise. All group and telephone coaching sessions will be audio recorded, and each month a random sample of $10 \%$ of the recordings will be coded by study staff to evaluate treatment fidelity. If fidelity falls below $90 \%$ on any of the fidelity checks, staff will be retrained.

\section{Outcome measures}

Assessments will be completed at baseline, 3-, 6-, and 12 -months. Participants will receive $\$ 40$ for completing each of the 3-, 6, and 12-month assessments. The procedures and measures are described below:

\section{Height, weight, BMI, and waist circumference}

Height will be measured using a wall-mounted stadiometer to the nearest $0.01 \mathrm{~cm}$. Body weight will be measured without shoes, wearing light clothing on a calibrated beam balance scale to the nearest $0.01 \mathrm{~kg}$. BMI $\left(\mathrm{kg} / \mathrm{m}^{2}\right)$ will be calculated using height and weight measurements. Waist circumference will be assessed by positioning an anthropometric tape midway between the palpated iliac crest and the palpated lowest rib margin in the mid-axillary lines. Two measurements will be taken and the mean value of each site will be used.

\section{Dietary intake adherence}

Dietary intake will be assessed at baseline, 3-, and 6-months via interview by a non-intervention registered dietician using the computer-assisted telephone interview (CATI) 24 hour recalls [37] using the multiple pass method to assess calorie and fat intakes. At each time point, three days (one weekend and two weekdays) will be randomly selected over a three week period to conduct the recalls. The data derived from the recalls will be analyzed using the annually updated University of Minnesota Nutrition Coordinating Center's (NCC) $\mathrm{Nu}$ trition Data System for Research (NDS-R-2005). Only STND and TECH will complete dietary recalls at 3- and 6-months.

\section{Physical activity adherence}

Physical activity will be objectively measured by an accelerometer (Actigraph \#7164, Pensacola, FL) over 7 days at baseline, 3-, and 6-months. The accelerometer will be attached to an elastic belt and worn on the right hip. Minutes of moderate-to-vigorous intensity physical activity will be measured in a modified bout lasting a minimum of eight consecutive minutes. Adherence will be ascertained on the basis of how well the participant's bout-corrected accelerometer count corresponds to the weekly activity goal.

\section{Dietary self-monitoring adherence}

Dietary self-monitoring adherence will be assessed as the number of days reporting dietary intake on the paper Keeping Track diaries (STND and SELF) or smartphone application $(\mathrm{TECH})$. In order to receive full adherence credit for a day of self-monitoring, participants must have $\geq 1000$ calories recorded and $\geq 25$ grams of fat. 


\section{Physical activity self-monitoring adherence}

For STND and SELF, physical activity self-monitoring adherence will be assessed as the number of days reporting physical activity in the paper Keeping Track diaries, divided by the number of possible days. For TECH participants, self-monitoring adherence will be operationalized as the number of days the accelerometer was worn $\geq 8$ hours, divided by the number of possible days.

\section{Coaching time}

Coaches will monitor the number of minutes preparing for and conducting individual coaching calls throughout the first 6 months of the intervention.

\section{Data analytic plan}

\section{Linear mixed modeling}

Data will be analyzed on an intent-to-treat basis. The basic modeling approach will entail linear mixed models for longitudinal data. The primary aim is to test for treatment group differences on weight loss at 3-, 6-, and 12-months as well as differences in behavioral adherence and goal attainment at 3- and 6-months. Linear mixed models do not require that participants are measured at all time points, and therefore can include participants with missing data across time. In these analyses, we will examine changes across time by treating time as a nominal variable in the analysis to allow for non-linear trends. In addition to the effect of time, the main independent variable is treatment groups, a between-subjects factor with 3 levels: STND, TECH and SELF. A priori Helmert contrasts [38] will be used to test the following comparisons at 3- and 6-months: 1) STND and TECH vs. SELF and 2) TECH vs. STND. We will also examine group by time interactions to test whether change in outcome across time differs by group.

\section{Mediation analysis}

We hypothesize that the weight loss advantage for those assigned to TECH would be explained (entirely or partially) by TECH's higher rates of adherence to selfmonitoring and attainment of behavioral goals. To test this mediation hypothesis, we will fit a linear mixed model predicting weight loss similar to the model described above, but now including in the regression equation the potential mediating variables corresponding to adherence to self-monitoring. To the extent that adherence mediate the effects of the experimental condition, in the model with adherence included, we expect the coefficient on the treatment group by time interaction variable to be reduced compared to the treatment group by time interaction coefficient from the model without adherence. This difference provides an estimate of the extent to which adherence may mediate the effects of the experimental condition on weight loss.
Bootstrapping will be used to estimate the sampling variability of the mediation effect.

\section{Sample size and power}

We will randomize $n=32$ participants to each treatment group and assume $6 \%$ of participants will drop out by the end of the study so that there will be $n=30$ participants in each group at the final endpoint. The power calculations below are based on the second Helmert contrast: TECH vs. STND because this contrast contains the fewest number of subjects (30 in each group) and we expect this contrast to have the smallest effect sizes. The first Helmert contrast: STND and TECH vs. SELF has a larger sample size (60 vs. 30) and we anticipate larger effects sizes in this contrast due to the limited intensity of the SELF intervention. Therefore, by powering the study based on the second contrast, we will have more than adequate power for the first contrast.

\section{Weight loss}

We measure weight loss at 3, 6, and 12 months. Using data from a previous weight loss trial [16], we saw standard deviations of 8.4 and 10.7 pounds at months 3 and 6 , respectively, with a correlation of 0.86 between the two time points. With $80 \%$ power and an $n=30$ in both groups, we will be able to detect a difference of 6.6 pounds between the two groups.

\section{Diet self-monitoring adherence}

We measure dietary self-monitoring adherence at 3 and 6 months as the ratio of actual days of diet selfmonitoring relative to the possible number of days. Using data from a previous trial [16], we observed a standard deviation of 0.31 and 0.34 at 3 and 6 months, respectively, as well as a correlation of 0.93 between the two time points. Thus, with $80 \%$ power and an $n=30$ in both groups, we will be able to detect a difference in adherence ratios of 0.23 between the two groups.

\section{Physical activity self-monitoring adherence}

Physical activity self-monitoring will be assessed as the number of days reporting physical activity on paper diaries (STND and SELF) or wearing the accelerometer (TECH), divided by the number of possible days. Data from a previous trial [16] shows a standard deviation of 0.30 at 3 months and 0.33 at 6 months and a correlation of 0.91 between the two time points. With $80 \%$ power and an $\mathrm{n}=30$ in both groups, we will be able to detect a difference in percent adherence of $22 \%$ between the two groups.

\section{Dietary intake adherence}

We measure adherence to calorie goals at 3 and 6 months using 3 day 24 hour recalls. Using pilot data 
from a previous trial, we observed a standard deviation of change in calories of 498 at month 3 and 431 at month 6, with a correlation of 0.9 between the two time points. With $80 \%$ power and an $n=30$ in both groups, we will be able to detect a calorie reduction difference of 375 calories.

\section{Physical activity adherence}

Physical activity adherence is measured by accelerometer at months 3 and 6 as the number of minutes of moderate to vigorous intensity physical activity per week. Using data from a previous trial [39], we assume a standard deviation of 142 minutes/week at months 3 and 6, with a correlation of 0.9 between the two time points. With $80 \%$ power and an $n=30$ in both groups, we will be able to detect a 100 minute/week difference between the two groups.

\section{Discussion}

Obesity continues to be a significant public health issue worldwide and guidelines recommend intensive behavioral interventions for all obese adults $[1,2,40]$. However, efficacious and scalable interventions have not yet been widely implemented. Mobile technology provides an opportunity to develop interventions with lower costs, less burden, and a greater reach, particularly as mobile usage rates continue to rise. As of February 2012, approximately $46 \%$ of American adults currently own a smartphone, an increase from 36\% in May 2011 [26]. Minority groups, including Blacks and Hispanics saw an increase in smartphone use from $44 \%$ to $49 \%$ during this same time period [26]. Smartphones have the ability to extend key components of intensive weight loss treatment into real-world settings, while reducing treatment costs and maintaining efficacious weight loss outcomes. The ENGAGED study is an innovative randomized controlled trial that will examine the feasibility and efficacy of a theory-guided, technology-supported weight loss program. Specifically, a total of 96 obese adults will be randomized to one of three conditions: a) Standard behavioral weight loss program (STND); b) technologysupported behavioral weight loss program (TECH); or c) self-guided behavioral weight loss program (SELF). Differences in weight loss, behavioral adherence, and goal attainment will be examined between groups.

The technology-supported condition will utilize a smartphone application designed to target known health behavior change mechanisms. Participants will be able to track weight and dietary intake, receive real-time feedback on behaviors, and communicate with team members by using a private message board and direct messaging. The application will also connect wirelessly via Blue-tooth to an accelerometer to provide real-time feedback on objectively measured physical activity. The
ENGAGED technology will enable participants to experience ongoing support and accountability by allowing study coaches to continuously view participant's up-todate data, enabling them to provide timely support in the event of technical difficulties and tailored, immediate feedback during coaching calls.

Limited research has been conducted on smartphonesupported weight loss interventions, leaving numerous unanswered questions and directions for future research. Future studies are especially needed on the efficacy of mobile health interventions in underrepresented groups (i.e., older adults, ethnic minorities, uneducated, and low SES). Also needed are studies on the efficacy of technology supported interventions over the long-term, as weight loss maintenance continues to be one of the greatest challenges to obesity interventions [41]. To date, there are few or no behavioral interventions that fully maintain weight losses after treatment ends [42]. If the ENGAGED study provides preliminary evidence that a smartphone-supported intervention improves short-term weight loss and adherence outcomes, further studies will be warranted to examine whether the use of this technology can enhance long-term weight loss maintenance.

The ENGAGED Study is a theory-guided randomized controlled trial that will examine the efficacy and feasibility of a smartphone-supported implementation of the DPP on weight loss and behavioral adherence. Although smartphone usage is on the rise, the evidence base evaluating applications for health behavior change is extremely sparse. This study will make an important contribution by rigorously examining the effect of a novel, scalable smartphone technology system on self-monitoring adherence and weight loss outcomes.

\section{Abbreviations}

CST: Control systems theory; DPP: Diabetes prevention program; SELF: Selfguided behavioral weight loss; STND: Standard behavioral weight loss; TECH: Technology-supported behavioral weight loss.

\section{Competing interests}

The authors declare no competing interests.

\section{Authors' contributions}

$B S, S P, A M$, and JS were responsible for study conceptualization, design, and developing the analytic plan. $C P, J D, J B, A S, A D$, and $A P$ participated in operationalizing the intervention. $C P, J D, A M, J B$, and $B S$ drafted the manuscript. All authors read and approved the final manuscript.

\section{Acknowledgements}

This project is supported by Award Number RC1DK087126 from the National Institute of Diabetes and Digestive and Kidney Diseases. The content is solely the responsibility of the authors and does not necessarily represent the official views of the National Institute of Diabetes and Digestive and Kidney Diseases or the National Institutes of Health. The authors thank Megan Roehrig and Stephanie Russell for their initial insight into the study.

\section{Author details}

${ }^{1}$ Current Address: Department of Preventive Medicine, Feinberg School of Medicine, Northwestern University, 680 N. Lake Shore Drive, Suite 1400, Chicago, IL 60611, USA. ${ }^{2}$ Department of Medicine, Division of Preventive and 
Behavioral Medicine, University of Massachusetts Medical School, 55 Lake Avenue North, Worcester, MA 01655, USA.

Received: 12 October 2012 Accepted: 27 November 2012 Published: 30 November 2012

\section{References}

1. Flegal KM, Carroll MD, Kit BK, Ogden CL: Prevalence of obesity and trends in the distribution of body mass index among US adults, 1999-2010. JAMA 2012, 307(5):491-497.

2. WHO: Obesity. http://www.who.int/topics/obesity/en/

3. Resnick HE, Valsania P, Halter JB, Lin X: Relation of weight gain and weight loss on subsequent diabetes risk in overweight adults. $J$ Epidemiol Community Health 2000, 54(8):596-602.

4. Mokdad AH, Marks JS, Stroup DF, Gerberding JL: Actual causes of death in the United States, 2000. JAMA 2004, 291(10):1238-1245.

5. Must A, Spadano J, Coakley EH, Field AE, Colditz G, Dietz WH: The disease burden associated with overweight and obesity. JAMA 1999, 282(16):1523-1529.

6. Bianchini $\mathrm{F}$, Kaaks $\mathrm{R}$, Vainio $\mathrm{H}$ : Overweight, obesity, and cancer risk. Lancet Oncol 2002, 3(9):565-574

7. Finkelstein EA, Trogdon JG, Cohen JW, Dietz W: Annual medical spending attributable to obesity: payer- and service-specific estimates. Health Aff 2009, 28(5):W822-W831.

8. Knowler WC, Barrett-Connor E, Fowler SE, Hamman RF, Lachin JM, Walker EA, Nathan DM: Reduction in the incidence of type 2 diabetes with lifestyle intervention or metformin. N Engl J Med 2002, 346(6):393-403.

9. Eddy DM, Schlessinger L, Kahn R: Clinical outcomes and cost-effectiveness of strategies for managing people at high risk for diabetes. Ann Intern Med 2005, 143(4):251-264.

10. Whittemore R, Melkus G, Wagner J, Dziura J, Northrup V, Grey M: Translating the diabetes prevention program to primary care: a pilot study. Nurs Res 2009, 58(1):2-12.

11. Ackermann RT, Finch EA, Brizendine E, Zhou HH, Marrero DG: Translating the diabetes prevention program into the community - the DEPLOY pilot study. Am J Prev Med 2008, 35(4):357-363.

12. Katula JA, Vitolins MZ, Rosenberger EL, Blackwell CS, Morgan TM, Lawlor MS, Goff DC Jr: One-year results of a community-based translation of the diabetes prevention program: healthy-living partnerships to prevent diabetes (HELP PD) project. Diabetes Care 2011, 34(7):1451-1457.

13. Cramer JS, Sibley RF, Bartlett DP, Kahn LS, Loffredo L: An adaptation of the diabetes prevention program for use with high-risk, minority patients with type 2 diabetes. Diabetes Educ 2007, 33(3):503-508.

14. Coons MJ, DeMott A, Buscemi J, Duncan JM, Pellegrini CA, Steglitz J, Pictor A, Spring B: Technology interventions to curb obesity: a systematic review of the current literature. Curr Cardiovasc Risk Rep 2012, 6:120-134.

15. Burke LE, Styn MA, Sereika SM, Conroy MB, Ye L, Glanz K, Sevick MA, Ewing $\sqcup$ : Using mHealth technology to enhance self-monitoring for weight loss: a randomized trial. Am J Prev Med 2012, 43(1):20-26.

16. Spring B, Duncan JM, Janke EA, Kozak AT, McFadden HG, Demott A, Pictor A, Epstein LH, Siddique J, Pellegrini CA, et al: Integrating technology into standard weight loss treatment: a randomized controlled trial. Arch Intern Med. in press.

17. Patrick K, Raab F, Adams MA, Dillon L, Zabinski M, Rock CL, Griswold WG, Norman GJ: A text message-based intervention for weight loss: randomized controlled trial. J Med Internet Res 2009, 11(1):e1.

18. Haapala I, Barengo NC, Biggs S, Surakka L, Manninen P: Weight loss by mobile phone: a 1-year effectiveness study. Public Health Nutr 2009, 12(12):2382-2391.

19. Pellegrini CA, Verba SD, Otto AD, Helsel DL, Davis KK, Jakicic JM: The comparison of a technology-based system and an in-person behavioral weight loss intervention. Obesity (Silver Spring) 2012, 20(2):356-363.

20. Shuger SL, Barry WW, Sui X, McClain A, Hand GA, Wilcox S, Meriwether RA, Hardin JW, Blair SN: Electronic feedback in a diet- and physical activitybased lifestyle intervention for weight loss: a randomized controlled trial. Int J Behav Nutr Phys Act 2011, 8:41.

21. Neve M, Morgan PJ, Jones PR, Collins CE: Effectiveness of web-based interventions in achieving weight loss and weight loss maintenance in overweight and obese adults: a systematic review with meta-analysis. Obes Rev 2010, 11(4):306-321.
22. Saperstein SL, Atkinson NL, Gold RS: The impact of Internet use for weight loss. Obes Rev 2007, 8(5):459-465.

23. Tate DF, Jackvony EH, Wing RR: Effects of Internet behavioral counseling on weight loss in adults at risk for type 2 diabetes - A randomized trial. JAMA 2003, 289(14):1833-1836.

24. Tate DF, Jackvony EH, Wing RR: A randomized trial comparing human e-mail counseling, computer-automated tailored counseling, and no counseling in an Internet weight loss program. Arch Intern Med 2006, 166(15):1620-1625.

25. Patrick K, Griswold WG, Raab F, Intille SS: Health and the mobile phone. Am J Prev Med 2008, 35(2):177-181.

26. Pew internet \& American life project: Smartphone update. $2012 \mathrm{http}: / /$ pewinternet.org/Reports/2012/Smartphone-Update-2012.aspx.

27. Carver C, Scheier M: On the self regulation of behavior. New York: Cambridge University Press; 1998.

28. Christakis NA, Fowler $\mathrm{JH}$ : The spread of obesity in a large social network over 32 years. N Engl J Med 2007, 357(4):370-379.

29. Wing RR, Jeffery RW: Benefits of recruiting participants with friends and increasing social support for weight loss and maintenance. J Consult Clin Psychol 1999, 67(1):132-138.

30. Krukowski RA, Harvey-Berino J, Ashikaga T, Thomas CS, Micco N: Internetbased weight control: the relationship between web features and weight loss. Telemed J e Health 2008, 14(8):775-782.

31. Goldberg JH, Kiernan M: Innovative techniques to address retention in a behavioral weight-loss trial. Health Educ Res 2005, 20(4):439-447.

32. Program TDP: Design and methods for a clinical trial in the prevention of type 2 diabetes. Diabetes Care 1999, 22(4):623-634

33. Netzer CT: The complete book of food counts. 7th edition. New York: Dell Pub; 2006

34. Ryan DH, Espeland MA, Foster GD, Haffner SM, Hubbard VS, Johnson KC, Kahn SE, Knowler WC, Yanovski SZ: Look AHEAD (Action for Health in Diabetes): design and methods for a clinical trial of weight loss for the prevention of cardiovascular disease in type 2 diabetes. Control Clin Trials 2003, 24(5):610-628.

35. Renjilian DA, Perri MG, Nezu AM, McKelvey WF, Shermer RL, Anton SD: Individual versus group therapy for obesity: effects of matching participants to their treatment preferences. J Consult Clin Psychol 2001, 69(4):717-721

36. Kramer MK, Kriska AM, Venditti EM, Miller RG, Brooks MM, Burke LE, Siminerio LM, Solano FX, Orchard TJ: Translating the diabetes prevention program: a comprehensive model for prevention training and program delivery. Am J Prev Med 2009, 37(6):505-511.

37. Posner BM, Borman CL, Morgan JL, Borden WS, Ohls JC: The validity of a telephone-administered 24-hour dietary recall methodology. Am J Clin Nutr 1982, 36(3):546-553

38. Bock RD: Multivariate statistical methods in behavioral research. New York: Mc-Graw Hill; 1975

39. Spring B, Schneider K, McFadden HG, Vaughn J, Kozak AT, Smith M, Moller AC, Epstein LH, Demott A, Hedeker D, et al: Multiple behavior changes in diet and activity: a randomized controlled trial using mobile technologybehavior changes in diet and Activity. Arch Intern Med 2012, 172(10):789-796.

40. USPSTF: ecommendations - Obesity in adults. http://www. uspreventiveservicestaskforce.org/recommendations.htm.

41. Elfhag K, Rossner S: Who succeeds in maintaining weight loss? A conceptual review of factors associated with weight loss maintenance and weight regain. Obes Rev 2005, 6(1):67-85.

42. Svetkey LP, Stevens VJ, Brantley PJ, Appel LJ, Hollis JF, Loria CM, Vollmer WM, Gullion CM, Funk K, Smith P, et al: Comparison of strategies for sustaining weight loss: the weight loss maintenance randomized controlled trial. JAMA 2008, 299(10):1139-1148.

\section{doi:10.1186/1471-2458-12-1041}

Cite this article as: Pellegrini et al:: A smartphone-supported weight loss program: design of the ENGAGED randomized controlled trial. BMC Public Health 2012 12:1041. 